\title{
Development of a Neutron Energy-Biased In-Air Figure-of-Merit for Predicting In-Phantom BNCT Neutron Beam Characteristics
}

\author{
D.L. Bleuel, ${ }^{a, b}$ R.J. Donahue, ${ }^{b}$ B.A. Ludewigt, ${ }^{b}$ and J. Vujic ${ }^{a}$ \\ aDepartment of Nuclear Engineering \\ University of California, Berkeley \\ and \\ bAccelerator and Fusion Research Division \\ Ernest Orlando Lawrence Berkeley National Laboratory \\ University of California \\ Berkeley, California 94720
}

March 1998

This work was supported by the Director, Office of Energy Research, Nuclear Physics Division of the Office of High Energy and Nuclear Physics, of the U.S. Department of Energy under Contract No. DE-AC03-

76 SF00098. 


\section{DISCLAIMER}

This report was prepared as an account of work sponsored by an agency of the United States Government. Neither the United States Government nor any agency thereof, nor any of their employees, make any warranty, express or implied, or assumes any legal liability or responsibility for the accuracy, completeness, or usefulness of any information, apparatus, product, or process disclosed, or represents that its use would not infringe privately owned rights. Reference herein to any specific commercial product, process, or service by trade name, trademark, manufacturer, or otherwise does not necessarily constitute or imply its endorsement, recommendation, or favoring by the United States Government or any agency thereof. The views and opinions of authors expressed herein do not necessarily state or reflect those of the United States Government or any agency thereof. 


\section{DISCLAIMER}

Portions of this document may be illegible in electronic image products. Images are produced from the best available original document. 


\title{
Development of a Neutron Energy-Biased In-Air Figure-of-Merit for Predicting In-Phantom BNCT Neutron Beam Characteristics.*
}

\author{
D. L. Bleuel, ${ }^{a, b}$ R. J. Donahue, ${ }^{a}$ B. A. Ludewigt ${ }^{a}$, and J. Vujic ${ }^{b}$
}

${ }^{a}$ Ernest Orlando Lawrence Berkeley National Laboratory

${ }^{b}$ Department of Nuclear Engineering, University of California

Berkeley, CA 94720

\section{INTRODUCTION}

Maximizing the efficacy of a Boron Neutron Capture Therapy (BNCT) modality requires improvements in two areas: development of better boron-delivering drugs and better tailoring of the epithermal neutron beam. Previous attempts at the latter have not always been successful in predicting optimal in-phantom results, often assuming that all neutrons within a certain "useful" energy range are equally valuable for BNCT purposes. Some studies have recognized that higher energy epithermal neutrons $(\sim 10 \mathrm{keV})$ and more forward-directed neutrons provide more penetrating beams. This results in higher tumor doses at centerline phantom depths. The exact effect of neutron beam energy spectrum shaping for BNCT has

*This work was supported by the Director, Office of Energy Research, Nuclear Physics Division of the Office of High Energy and Nuclear Physics, of the U.S. Department of Energy under Contract No. DEAC03-76DF00098. 
not been thoroughly explored. One reason for this has been the usage of inappropriate and often misleading in-air figures-of-merit.

A few previous studies ${ }^{1}$ have examined the effects of monoenergetic neutron beams, but have not fully applied these results to help direct the design of an optimal neutron beam spectrum. In this report we examine the in-phantom dose characteristics of nearly monoenergetic neutron beams (with narrow energy bins) in the range from thermal to 14 $\mathrm{MeV}$, using the MCNP Monte Carlo code, and apply the results to predict the relative depthdose characteristics of BNCT neutron sources. By scrutinizing these beams on a per-neutron basis, the benefits and disadvantages of each beam's spectrum can be determined and used to guide future neutron spectrum development. Additionally, by computationally unfolding the energy-dependent dose responses with an input spectrum, in-phantom figures-of-merit may be applied successfully to an in-air spectrum. This procedure produces, in essence, an in-air figure-of-merit that predicts in-phantom beam characteristics.

\section{FIGURES-OF-MERIT}

Many different figures of merit have been used to evaluate the characteristics of neutron beams for BNCT. No one parameter has yet emerged clearly as the most appropriate. These can be grouped into two catagories: in-air figures-of-merit and in-phantom figures-of-merit. The most popular in-air figures-of-merit in the BNCT community appear to be $D_{f} / \Phi_{e p i}$ and $D_{\gamma} / \Phi_{e p i}$, which are the ratios of, respectively, the in-air fast dose and the in-air photon dose to the epithermal neutron flux.. Although popular, these parameters do very little towards evaluating the clinical effectiveness of a particular beam and there are no clear parameters on what values are acceptable. It is generally assumed that the higher these values, the better the beam. However, these figures-of-merit only pit the worth of a loosely-defined range of neutrons, called "epithermal," against the effects of the unwanted fast and gamma doses at the surface of the skin. They do not necessarily relate to the actual treatment doses, which are usually limited by a maximum normal tissue dose at some depth in the brain, not at the skin surface.

Ideally, a figure-of-merit should reflect the in-phantom dose that can be delivered to a tumor volume, either real or hypothetical, within clinically-defined limits such as the maximum allowable tissue dose and/or a skin dose limit. A correct evaluation involves in- 


\begin{tabular}{|l|l|l|l|}
\hline \multicolumn{2}{|c|}{ Figure-of-Merit } & \multicolumn{1}{|c|}{ BMRR } & \multicolumn{1}{c|}{ LBNL } \\
\hline \hline In-Air & $D_{f} / \Phi_{e p i}\left[\mathrm{~Gy} /\left(\mathrm{n} / \mathrm{cm}^{2}\right)\right]$ & $4.8 \times 10^{-13}$ & $8.9 \times 10^{-13}$ \\
& $D_{\gamma} / \Phi_{e p i}\left[\mathrm{~Gy} /\left(\mathrm{n} / \mathrm{cm}^{2}\right)\right]$ & $2.0 \times 10^{-13}$ & $3.2 \times 10^{-13}$ \\
\hline \multirow{5}{*}{ In-Phantom } & RBE Equiv. tumor dose (8cm) (Gy-Eq.) & 14.5 & 23.4 \\
& Advantage depth (cm) & 8.4 & 9.9 \\
& RBE Equiv. $D_{f}$ at $D_{\text {tis. }}$ (Max.) (Gy-Eq.) & 2.05 & 0.632 \\
\hline
\end{tabular}

Table 1: Figures-of-merit for two neutron beams. The LBNL beam has a higher fast dose inair than the BMRR beam, but a lower in-phantom fast dose contribution to $\mathrm{D}_{\text {tis. }}$ (Max.), as well as superior in-phantom qualities. Equivalent doses are calculated assuming a maximum normal tissue tolerence of 12.5 Gy-eq. and using BMRR-defined clinical RBEs and compound factors.

phantom analysis, which is often not used since it is computationally time consuming and the results of different studies may not be comparable unless each use exactly the same phantom geometry and boron-delivering drug parameters.

Table 1 shows how $D_{f} / \Phi_{e p i}$ and $D_{\gamma} / \Phi_{e p i}$ can be misleading predictors of in-phantom behavior. Two neutron sources are evaluated: the beam currently used in clinical trials at the Brookhaven Medical Research Reactor (BMRR), ${ }^{2}$ and an accelerator-based source designed at LBNL. The LBNL design is an improvement on previous ${ }^{3}$ designs, with modifications including a lead reflector, and a cylindrical moderator consisting of $28 \mathrm{~cm}$ of Fluental $(69 \%$ $\mathrm{AlF}_{3}, 30 \% \mathrm{Al}, 1 \% \mathrm{LiF}$ ) followed by $6 \mathrm{~cm}$ of Teflon. The neutron source for this design is the ${ }^{7} \mathrm{Li}(\mathrm{p}, \mathrm{n})$ reaction, utilizing $2.4 \mathrm{MeV}$ protons. The in-phantom figures-of-merit demonstrate the superiority of the more penetrating LBNL beam, but the in-air figures-of-merit predict the opposite. Examination of the depth-dose response in Figure 1 shows why this is true. While the LBNL beam has a significantly higher in-air fast dose than the BMRR beam, the neutrons that contribute to this dose have much lower energies than the neutrons in the BMRR beam. Thus, these neutrons are absorbed more quickly and actually contribute a lower dose component to the treatment-limiting maximum tissue dose than in the BMRR beam. Also, the more penetrating LBNL beam provides a deeper point of maximum tissue dose, where the fast component is a smaller fraction of the total dose. Therefore, these in-air figures-of-merit are of limited use in determining the quality of a particular neutron beam.

Pursuit of a more useful in-air figure-of-merit remains an elusive, yet highly desirable goal. 


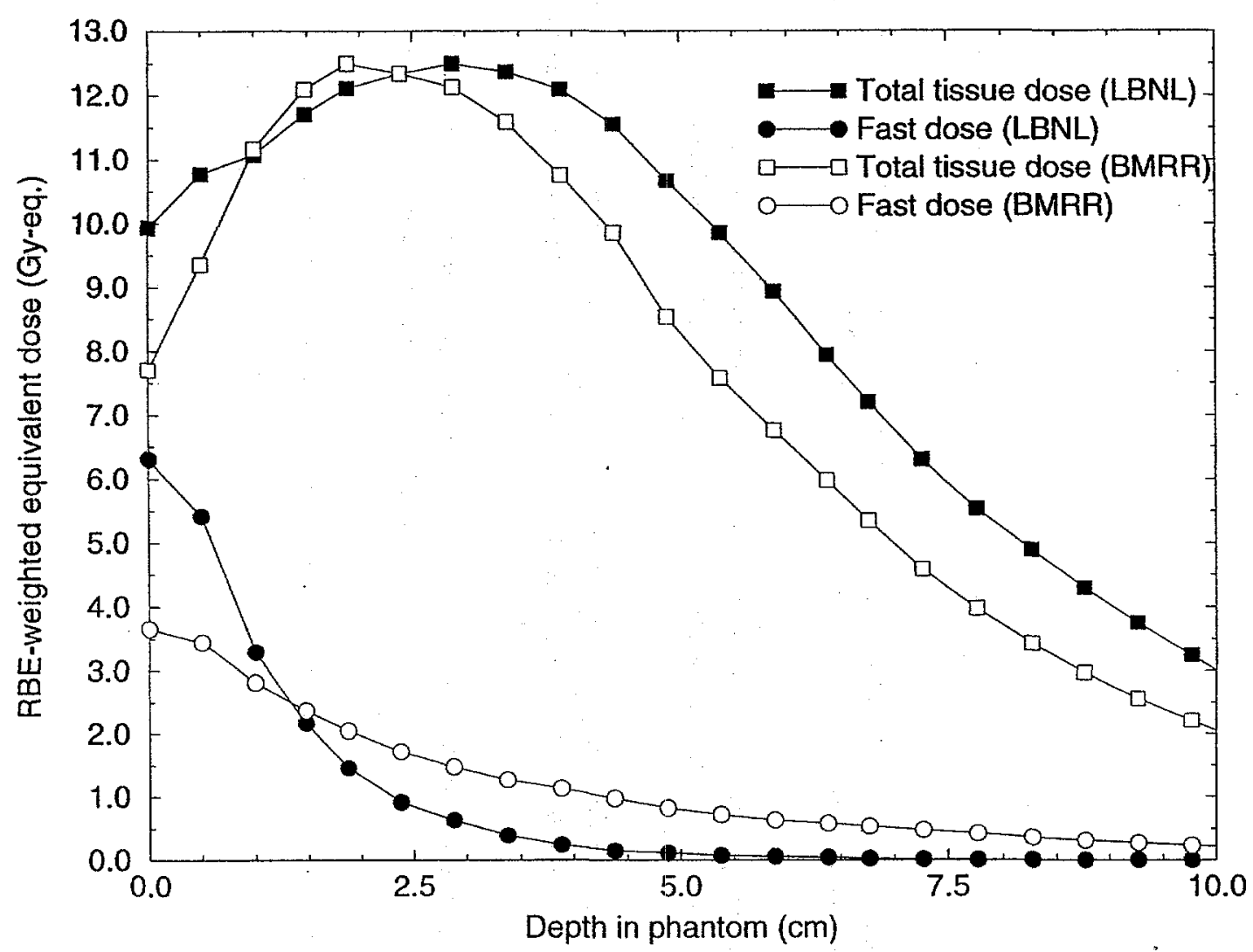

Figure 1: Total tissue dose response and fast dose component of BMRR and LBNL beams.

Since the determination of an in-phantom dose response often involves lengthy Monte Carlo simulations, much computer time can be saved if optimal beams could be predicted from the in-air neutron spectrum. This requires that an appropriate in-phantom figure-of-merit be identified to predict the clinical efficacy of in-air beams.

Our beam optimization goal has been to produce the most penetrating beam possible that delivers the highest tumor dose to a tumor at the center of the brain for fixed limits on maximum tissue dose and skin dose. The invasive, non-localized character of glioblastoma multiforme encourages the use of a whole-brain approach to treatment rather than limiting the treatment to variably defined tumor volumes. Because the center of the brain is the most difficult part to treat, virtually all cases benefit from maximizing the dose at this point. However, it is understood that certain easier-to-treat cases of extremely shallow and localized tumors may not require a maximally penetrating beam.

With these aims in mind, the most appropriate in-phantom figure-of-merit was chosen to be $\mathrm{TG}_{b m}$, the Therapeutic Gain at the brain midpoint, defined as the ratio of the RBE- 
weighted tumor dose at the brain midpoint to the maximum RBE-weighted tissue dose:

$$
\begin{gathered}
T G_{b m}=\frac{D_{t u m o r}(\text { Midpoint })}{D_{t i s .}(\text { Max. })} \\
D_{\text {tumor/tis. }}=C F_{\text {tumor } / t i s .} \cdot D_{B}+R B E_{N} \cdot D_{N}+R B E_{H} \cdot D_{H}+R B E_{\gamma} \cdot D_{\gamma}
\end{gathered}
$$

where $\mathrm{D}_{B}, \mathrm{D}_{N}, \mathrm{D}_{H}$, and $\mathrm{D}_{\gamma}$ refer respectively to the physical doses due to the ${ }^{10} \mathrm{~B}(\mathrm{n}, \alpha)$ boron, ${ }^{14} \mathrm{~N}(\mathrm{n}, \mathrm{p})$ nitrogen, ${ }^{1} \mathrm{H}\left(\mathrm{n}, \mathrm{n}^{\prime}\right)$ proton-recoil, and the $\gamma$ dose via the ${ }^{1} \mathrm{H}(\mathrm{n}, \gamma)$ capture reaction. The physical doses were multiplied by a corresponding compound factor (CF) or relative biological effectiveness (RBE). Boron concentrations and compound factors were used as established ${ }^{4}$ for the boron compound $p$-boronphenylalanine (BPA) along with RBEs as established in the dose calculation protocol for clinical trials at the BMRR: normal tissue ${ }^{10} \mathrm{~B}$ concentration: $13 \mathrm{ppm}$; normal tissue compound factor: 1.3 ; tumor ${ }^{10} \mathrm{~B}$ concentration: 45.5 ppm; tumor compound factor: 3.8; $\mathrm{RBE}_{N}: 3.2 ; \mathrm{RBE}_{H}: 3.2$; and $\mathrm{RBE}_{\gamma}: 1.0$. Because $\mathrm{TG}_{b m}$ is a ratio, it is independent of an actual defined limit on $\mathrm{D}_{t i s .}$ (Max.).

With $\mathrm{TG}_{b m}$ established as an appropriate in-phantom figure-of-merit for gauging beam quality, an in-air parameter can be sought to predict this quantity as a function of the neutron spectrum.

\section{NEUTRON ENERGY DOSE RESPONSE}

\subsection{Modeling}

The first step in predicting the dose response of any neutron energy spectrum was modeling a large number $(\sim 100)$ of nearly monoenergetic neutron energy distributions through a head phantom using the MCNP ${ }^{5}$ Monte Carlo code. Each simulation consisted of a monodirectional neutron beam with a narrow, uniform energy distribution, incident onto the full surface of a head phantom. A modified $S n y d e r^{6}$ head phantom was used, consisting of skin, bone, and brain in the geometry with surfaces described by the following equations:

$$
\left(\frac{X}{6.5}\right)^{2}+\left(\frac{Y}{6}\right)^{2}+\left(\frac{Z}{9}\right)^{2}=1
$$




$$
\begin{gathered}
\left(\frac{X+1}{8.3}\right)^{2}+\left(\frac{Y}{6.8}\right)^{2}+\left(\frac{Z}{9.8}\right)^{2}=1 \\
\left(\frac{X+1}{8.8}\right)^{2}+\left(\frac{Y}{7.3}\right)^{2}+\left(\frac{Z}{10.3}\right)^{2}=1
\end{gathered}
$$

The modification to the Snyder phantom was the addition of a half centimeter layer of skin as used by Liu et. al. ${ }^{7}$. Elemental compositions for brain, skin, and skull were taken from ICRU $46^{8}$. The midpoint of this phantom is at $7.3 \mathrm{~cm}$. Narrow energy bins were chosen instead of monoenergetic beams in order to assure that all energies between thermal and 14 $\mathrm{MeV}$ would be represented and that no resonances in material transport would be neglected or artificially dominate the response. The width of each bin was chosen to be equilogarithmic, with ten bins per energy decade.

For each simulation, the dose response for each reaction in Equation 2 was determined along the centerline of the beam through the brain. In addition, the dose responses for the oxygen and carbon neutron reactions were determined and added to Equation 1 . At very high neutron energies $(>\sim 1 \mathrm{MeV})$, the dose due to reactions with oxygen can approach nearly $10 \%$ that of the otherwise dominant proton recoil dose, due to resonances in oxygen's neutron cross section. While carbon's dose contribution is negligible, its dose response was added for completeness. For both these doses, an RBE of 3.2 was assumed.

In this fashion, a large database was constructed from which dose as a function of energy, position, element, and RBE can be determined for a wide parallel neutron beam. Using this database, the dose response of any input monodirectional neutron spectrum can be instantly calculated without the need for a separate Monte Carlo simulation. These input neutron spectra can be either realistically generated spectra or hypothetical beams.

Some effort was made to develop a simple algebraic representation of the dose response as a function of the above parameters through curve fitting. However, it was not possible to develop a simple equation without introducing large errors compared to the accuracy of the original dose response database. For this reason, this option is not used and all in-air neutron spectra are directly folded into the energy-dependent dose response database. This ensures the highest degree of accuracy. 


\subsection{Moderator Optimization}

This new method of evaluating an in-air neutron beam spectrum was initially tested by comparing the $\mathrm{TG}_{b m}$ prediction against that calculated with the INEEL Monte Carlo treatment planning code, BNCT RTPE. ${ }^{9}$ There are many differences in these two models, including elemental composition, phantom geometry, beam size, and angular distribution. Therefore, the values of $\mathrm{TG}_{b m}$ calculated with each method were expected to be quite different. However, each method should produce the same results in judging different neutron spectra against each other. Figure 2 shows that for two different moderators $\left(\mathrm{Al} / \mathrm{AlF}_{3}\right.$ and $\left.\mathrm{D}_{2} \mathrm{O}\right)$, the same thickness of moderator is predicted as optimal, independent of the model chosen. It should be noted that because the database includes data only from monodirectional neutrons, which are inherently more penetrating, it cannot be used to produce accurate absolute doses and can only be used to gauge different neutron spectra of similar $J / \Phi$ against each other. In particular, if there are additional constraints on treatment other than a maximum tissue dose (eg: surface dose or treatment time), great care must be taken to not misinterpret the results given by the database. For instance, previous LBNL optimization constraints have placed a limit of 10.0 Gy-eq on the surface dose, in addition to a maximum tissue dose constraint of 12.5 Gy-eq. In beams where BNCT_RTPE calculates a surface dose of $10.0 \mathrm{~Gy}$-eq, the database response is only 8.5 Gy-eq. This seems to be a consistent underrepresentation for beams with a typical $J / \Phi$ of $\sim 0.8$. This should be considered when a skin dose constraint is applied. To be fully useful in predicting dose responses, however, the database should be expanded to allow for angular dependence.

\subsection{The Ideal Neutron Spectrum}

Using a Monte Carlo code such as BNCT_RTPE or MCNP can take many hours or days of computer time, whereas evaluating an in-air neutron spectrum with the predetermined energy-dependant dose response database is instantaneous. Because the database allows instantaneous evaluation of a neutron energy spectrum, it becomes a powerful tool in determining the effect of changing existing neutron beams, or in quickly evaluating hypothetical neutron spectra.

This ability to instantly evaluate any input spectrum allows investigation into determining an "ideal" neutron spectrum for BNCT, which would produce the maximum value of 


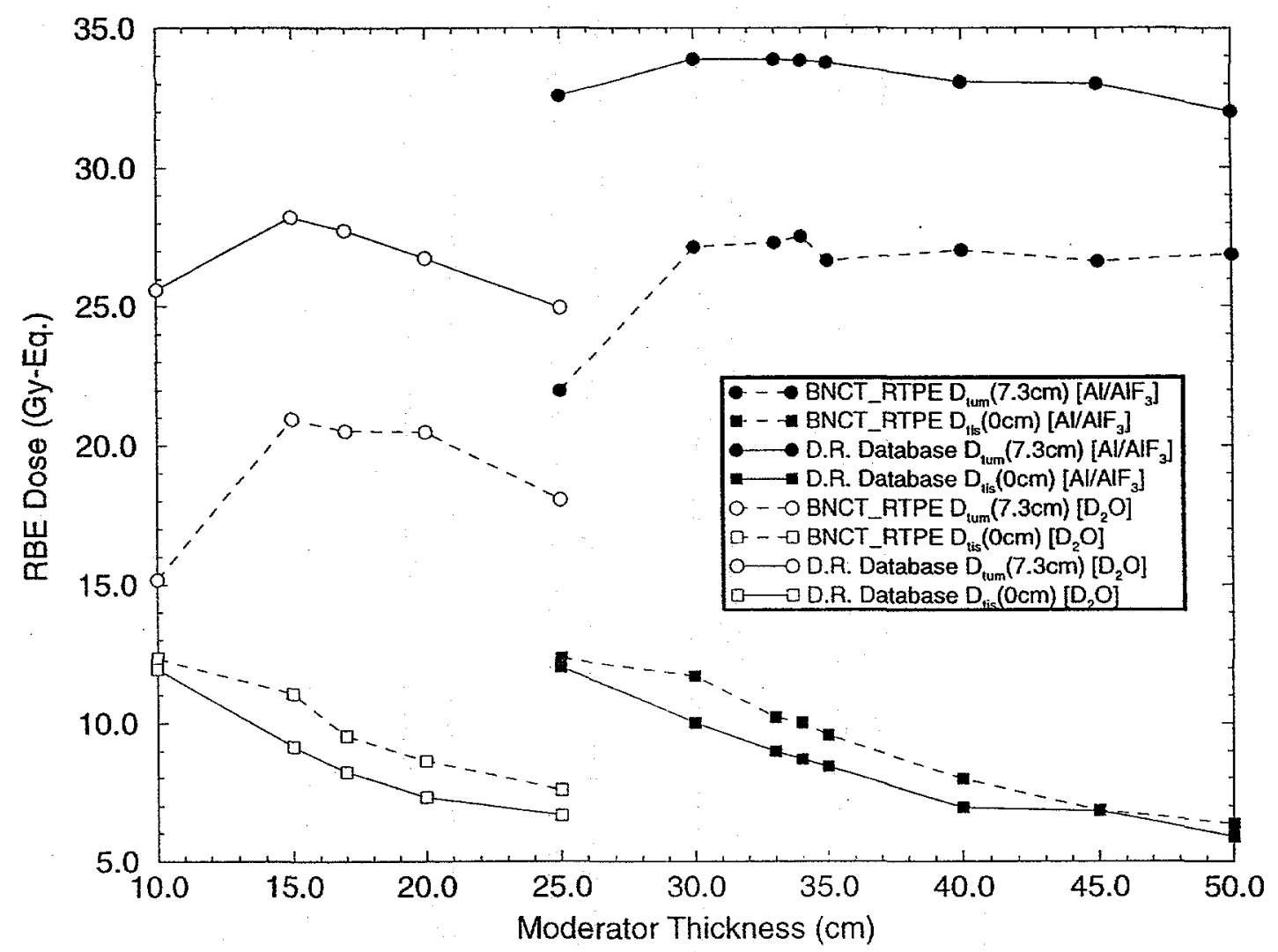

Figure 2: Comparison of using BNCT_RTPE or the Dose Response (D.R.) Database to optimize the moderator thickness of either $\mathrm{D}_{2} \mathrm{O}$ or $\mathrm{Al} / \mathrm{AlF}_{3}$. The maximum normal tissue tolerance was limited to $12.5 \mathrm{~Gy}$-Eq.

$\mathrm{TG}_{b m}$. Though such an "ideal" neutron spectrum may not be physically realizable, knowing its characteristics allows one to tailor real neutron beams to more closely match it. This can provide insight as to what gains may be made from further tailoring/filtering of existing beams and whether those gains are large enough to warrant corresponding losses in total neutron flux.

At first, it appears the realitive worth of a given neutron energy can be determined by simply plotting $\mathrm{TG}_{b m}$ as a function of neutron energy, as in Figure 3 . However, this is not necessarily a valid method of gauging the contribution of a given neutron energy to $\mathrm{TG}_{b m}$ in a real beam, which would contain a full spectrum of energies. $\mathrm{TG}_{b m}$ is dependent on the position of the point of maximum tissue dose, which can differ from beam to beam. In particular, for many monoenergetic beams, the point of maximum tissue dose may be at the skin surface. The contribution from this energy to the dose at the maximum tissue dose in 


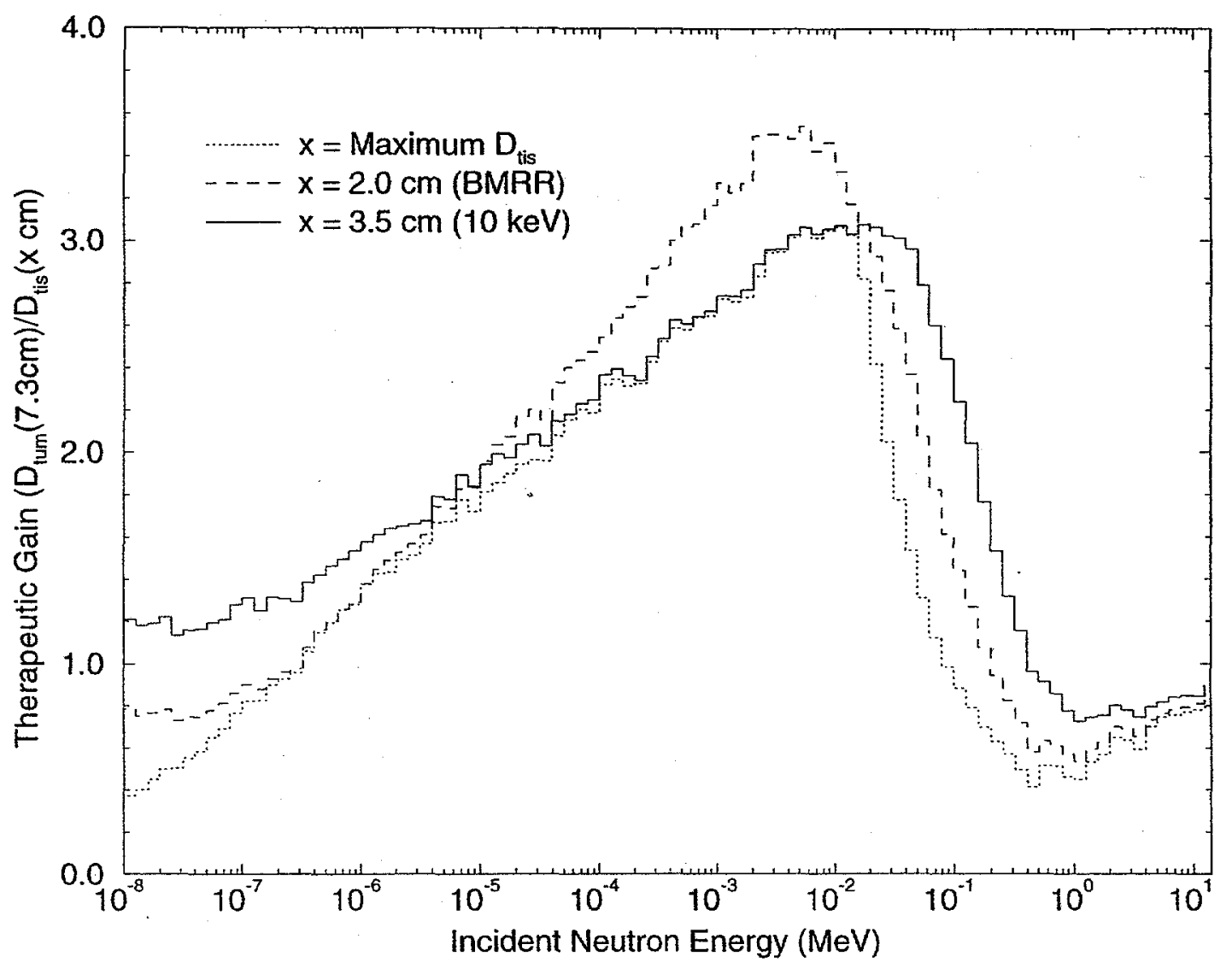

Figure 3: Therapeutic Gain at the brain midpoint as a function of neutron energy, representative of dose responses with peaks at $2.0 \mathrm{~cm}, 3.5 \mathrm{~cm}$, or where produced by the nearly monoenergetic beam.

a real beam, which may be at up to $3.5 \mathrm{~cm}$ depth, will be vastly different. This becomes problematic in that neutrons of a particular energy will be of different worth to different beams. For instance, $40 \mathrm{keV}$ neutrons might lower the $\mathrm{TG}_{b m}$ of a beam with a tissue dose maximum at $2.0 \mathrm{~cm}$, but the same energy neutrons may raise $\mathrm{TG}_{b m}$ in a beam with a dose maximum at $3.5 \mathrm{MeV}$.

Therefore, $\mathrm{TG}_{b m}(2.0 \mathrm{~cm})$ and $\mathrm{TG}_{b m}(3.5 \mathrm{~cm})$ have also been plotted in Figure 3. These values are defined as in Equation 1, with the exception that $\mathrm{D}_{t i s}(\mathrm{Max}$.) is assumed to be at $2.0 \mathrm{~cm}$ and $3.5 \mathrm{~cm}$, respectively. These values were chosen as they represent two extremes in maximum dose position. The BMRR tissue dose peaks at $2.0 \mathrm{~cm}$, while the maximum depth at which monodirectional beams peak are at $3.5 \mathrm{~cm}$. If the LBNL spectrum were monodirectional, it would peak close to $3.5 \mathrm{~cm}$. It's actual peak is at $2.9 \mathrm{~cm}$, the $\mathrm{TG}_{b m}$ curve for which looks similar to that of a $3.5 \mathrm{~cm}$ peak. By plotting $\mathrm{TG}_{b m}$ this way, the dose 


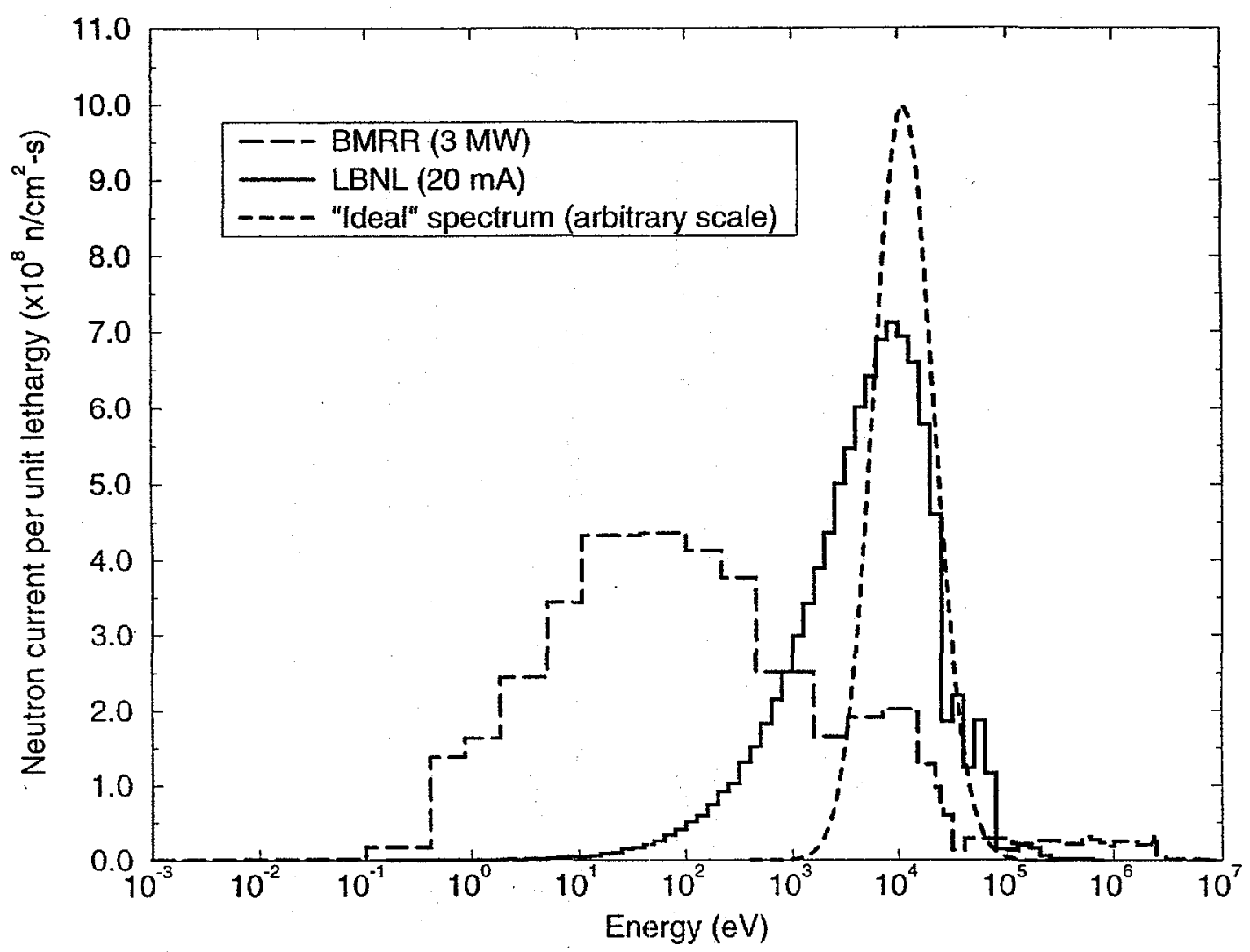

Figure 4: Neutron energy spectra for the BMRR beam, an LBNL accelerator-based design, and an ideal spectrum which produces the highest $\mathrm{TG}_{b m}$ possible.

contribution to different beams with different dose maxima for each neutron energy can be seen.

An obvious feature of Figure 3 is the maximum in $\mathrm{TG}_{b m}$, which occurs at around 10 $\mathrm{keV}$. In the most penetrating neutron beams $(\mathrm{Max} .=3.5 \mathrm{~cm})$, this maximum extends from about $4 \mathrm{keV}$ to about $40 \mathrm{keV}$. However, it never rises any higher. It was thought that certain higher energy neutrons ( $\sim 40 \mathrm{keV}-100 \mathrm{keV}$ ) might actually provide higher penetrability to a neutron beam and therefore be advantageous in small quantities, so long as there are not too many to adversely contribute to the point of maximum tissue dose or change its position. However, the fact that the $3.5 \mathrm{~cm}$ line never rises above its maximum at around $10 \mathrm{keV}$ suggests that $\mathrm{TG}_{b m}$ can never be increased above this value of about 3 . A $10 \mathrm{keV}$ neutron beam yields the highest $\mathrm{TG}_{b m}$ obtainable, though some width in energy can be tolerated with no loss in quality.

Also notable is the lower energy maximum in the $2.0 \mathrm{~cm}$ line, which rises above the 
maximum in the $3.5 \mathrm{~cm}$ line. Because these energies individually have $\mathrm{TG}_{b m}$ maxima at 3.5 $\mathrm{cm}$, their contribution to the dose at $2.0 \mathrm{~cm}$ is lower. It is nearly impossible, however, to conceive of a neutron spectrum which is composed primarily of neutrons at this energy, yet which has a maximum at $2.0 \mathrm{~cm}$ and this artifact cannot be exploited to produce a higher $\mathrm{TG}_{b m}$ than 3 .

The ideal neutron spectrum for BNCT is therefore shown, as in Figure 4, to be a spread of energies between $\sim 4 \mathrm{keV}$ and $\sim 40 \mathrm{keV}$, so long as the number of neutrons above $\sim 20$ $\mathrm{keV}$ is kept low enough to not adversely affect the position of $D_{\text {tis }}$ (Max.). Also shown are the BMRR beam and the LBNL accelerator-produced Fluental/Teflon-moderated beam. The LBNL beam is quite close to the ideal spectrum, demonstrating that there are few advantages to be gained by further alteration of the energy spectrum. The dose response database predicts that the BMRR, LBNL, and ideal spectra yield, respectively, a $\mathrm{TG}_{b m}$ of 2.0 , 2.9 and 3.0. Again, these values assume that each spectrum is made purely monodirectional and only reflect the relative differences in energy spectrum.

\section{CONCLUUSIONS}

This study has demonstrated the strong importance of the shape of the neutron energy spectrum for usage in $\mathrm{BNCT}$, which we have taken advantage of in shaping a nearly ideal spectrum. Our accelerator-based spectrum produces a monodirectional $\mathrm{TG}_{b m}$ that is within

$5 \%$ of the maximum that can be realized, leading us to conclude that future beam shaping should focus on improving directionality and beam intensity. The dose response database has proven a valuable tool in instantaneous evaluation of an in-air spectrum to predict inphantom parameters. However, to be fully applicable, it must be expanded to account for the angular distribution of a neutron beam. It would also benefit from expansion to allow for variable beam sizes and phantom geometries, after which it can be distributed for public use in optimizing neutron beam designs. 


\section{REFERENCES}

\section{References}

[1] Yanch, J. C., Zhou, X-1., Brownell, G. L., "A Monte Carlo Investigation of the Dosimetric Properties of Monoenergetic Neutron Beams for Neutron Capture Therapy," Radiation Research 126, 1-20 (1991).

[2] Coderre, J. A., Bergland, R., Capala, J., Chadha, M., Chanana, A. J., Elowitz, E., Joel, D. D., Liu, H. B., Slatkin, D., "Boron Neutron Capture Therapy for Glioblastoma Multiforme using $p$-boronophenylalanine and Epithermal Neutrons-Trials Design and Early Clinical Results," J. Neuro. Oncol. 33, 93-104 (1997).

[3] Bleuel, D. L., Donahue, R. J., Ludewigt, B. A., and Vujic, J., "Designing Acceleratorbased Epithermal Neutron Beams for Boron Neutron Capture Therapy," Medical Physics 25 (9), 1725-1734 (1998).

[4] Chanana, A. D., "Boron Neutron-Capture Therapy of Glioblastoma Multiforme at the Brookhaven Medical Research Reactor, A Phase I/II Study, FDA IND \#43,317, Protocol \#4," Med. Dep. Brookhaven Natl. Lab., Upton, NY 11973-5000, January, 1996.

[5] Briesmeister, J. F., MCNP - A General Monte Carlo N-Particle Transport Code, Version $4 B$, Los Alamos National Lab, 1997.

[6] Snyder, W. S., Fisher, Jr., H. L., Ford, M. R., Warner, G. G., "Estimates for Absorbed Fractions for Monoenergetic Photon Sources Uniformly Distributed in Various Organs of a Heterogeneous Phantom (Appendix B)," J. Nucl. Medicine, MIRD Supplement No. 3, Pamphlet 5 (1969).

[7] Liu, H. B., Greenberg, D. D., Capala, J., "An Improved Neutron Collimator for Brain Tumor Irradiations in Clinical Boron Neutron Capture Therapy," Medical Physics 23 (12), 2051-2061 (1996).

[8] "Photon, Electron, Proton and Neutron Interaction Data for Body Tissues," ICRU Report 46, February 28 (1992). 
[9] Nigg, D. W., Wheeler, F. J., Wessol, D. E., Capala, J., Chada, M., "Computational Dosimetry adn Treatment Planning for Boron Neutron Capture Therapy," J. NeuroOncol., 33, 93-104 (1997). 